\title{
Herringbone Pattern and CH- $\pi$ Bonding in the Crystal Architecture of Linear Polycyclic Aromatic Hydrocarbons
}

\author{
Albert Guijarro *† José A. Vergés ${ }^{*} \quad$ Emilio San-Fabián đ\$ \\ Guillermo Chiappe $\| \S \quad$ Enrique Louis $\| \S$
}

November 30, 2016

\section{Abstract}

The herringbone pattern is a pervasive structural motive found in most molecular crystals involving aromatic compounds. A plot of the experimental sublimation enthalpies of members of increasing size of the acene, phenacene and p-phenyl families versus the number of carbons uncovers a linear relationship between the two magnitudes, suggesting a major role of $\mathrm{CH}-\pi$ bonding. In this work we undertake the task of evaluating the relevance of the edge-to-face interaction (or $\mathrm{CH}-\pi$ bond) in the overall reticular energy of the crystal, in order to assess quantitatively the importance of this structural element. Following a heuristic approach, we considered the series of acenes, phenacenes and p-phenyls and analyzed the edge-to-face interaction between the molecules as they occur in the experimental crystal network. Isolation of the relevant molecular dimers allows to incorporate some of the most sophisticated tools of quantum chemistry and get a reliable picture of the isolated bond. When compared to the experimental sublimation energy, our results are conclusive: this sole interaction is the largest contribution to the lattice energy, and definitively dictates the crystal architecture in all the studied cases. Elusive enough, the edge-to-face interaction is mainly dominated by correlation interactions, specifically in the form of dispersion and, to a less extent, of

*corresponding author

†Departamento de Química Orgánica and Instituto Universitario de Síntesis Orgánica, Unidad Asociada del CSIC, Universidad de Alicante, San Vicente del Raspeig, 03690 Alicante, Spain.

${ }^{\ddagger}$ Departamento de Teoría y Simulación de Materiales, Instituto de Ciencia de Materiales de Madrid (CSIC), Cantoblanco, 28049 Madrid, Spain.

$\S$ Unidad Asociada del CSIC and Instituto Universitario de Materiales, Universidad de Alicante, San Vicente del Raspeig, 03690 Alicante, Spain.

`Departamento de Química Física, Universidad de Alicante, San Vicente del Raspeig, 03690 Alicante, Spain.

"Departamento de Física Aplicada, Universidad de Alicante, San Vicente del Raspeig, 03690 Alicante, Spain. 
charge transfer terms. A suggestive picture of the bond has been obtained by displaying the differences in local electron densities calculated by either correlated or non-correlated methods.

\section{Introduction}

\section{Face-to-face $(\pi-\pi)$ and edge-to-face $(\mathrm{CH}-\pi)$ bonding in PAH crystals}

Weak intermolecular chemical interactions play key roles in crystal engineering, biomolecular systems, molecular recognition, functional materials, supramolecular chemistry and a large number of diverse applications related to those ${ }^{1}$. The fundamental origin of some of these weak forces and its precise quantification is however difficult to assess, although numerous advances in quantum mechanics and useful tools for dissecting their outputs are now at our disposal $^{2-4}$. In that sense, polycyclic aromatic hydrocarbons (PAHs) represent a suitable model of study with certain advantages. They are based on light atoms which simplify notably the calculations, there is a large structural variety of PAHs, enriching with their diversity every possible study, and most importantly, there is a large set of experimental data about them available in the scientific literature for comparison. In spite of that, the overall current state of affairs in this area is far from satisfactory. Devoid of polar groups, the importance of dispersion forces in the description of the aromatic hydrocarbon interactions that originate dimers has been emphasized in recent theoretical studies ${ }^{5,6}$. London dispersion forces are attractive forces exhibited by nonpolar molecules because of the correlated movements of the electrons in interacting molecules. Indeed, in these studies not only the face-to-face $(\pi-\pi)$ interaction, but also the edge-to-face (T-shaped) configuration of the benzene dimer are mainly of the dispersion type. This is somewhat awkward for the edge-to-face interaction since dispersion relies on the size and polarizability of the interacting surfaces $^{7}$, of which both are minimal at the edge of the molecule.

In general, calculations provide a scenario of different configurations coexisting due to small differences in energy among them for the smaller arene, i.e. benzene. These configurations have been referred to by using a variety of names, but in the essential the relevant ones can be classified into two main categories, a) those displaying face-to-face interactions, also called parallel, $\pi-\pi$, $\pi$-stacked or sandwich, and b) those with edge-to-face interactions, also called T-shaped, Y-shaped, $\sigma-\pi, \mathrm{H}-\pi$ or $\mathrm{CH}-\pi$, which can accommodate further useful descriptors such as displaced, shifted, slipped, offset, off-centre, displaced-rotated, crossed, etc. for a) group and tilted for b) group. For the first member of the series, benzene, there is a certain agreement confining the set of most likely configurations to the parallel displaced and the tilted T-shaped, both within a narrow energy margin. At the CCSD(T)/CBS limit ${ }^{8}$ , which is considered a reference for other calculations, the binding energy is ca. $2.8 \mathrm{kcal}$ $\mathrm{mol}^{-1}$ and the difference between configurations is only $\approx 0.1 \mathrm{kcal} \mathrm{mol}^{-1}$ favoring the tilted T-shaped $^{9-11}$. Many of the recently developed dispersion corrected DFT methods failed to provide even the right energy ordering for the benzene dimer configurations ${ }^{12}$, perhaps with some exceptions ${ }^{2}$, while the heavily parametrized functional M06-2X can reasonably repro- 
duce the energy difference but only at the CBS level ${ }^{12}$. RI-MP2/CBS seems to overestimate the dispersion energy and gives an overall minimum at the displaced parallel configuration ${ }^{9}$ - In general, although extensive studies have been done for the benzene dimer, there is no unified description of the factors that contribute to the pairing interactions ${ }^{13}$, in spite that there is some consensus on the experimental geometry in the gas phase towards an edge-to-face interaction, in the form of tilted T-shaped ${ }^{14}$.

As the size of the $\pi$ system increases (i.e., the number of rings), so do the dispersion forces and with them the prevalence for face-to-face structures because it allows for larger surfaces of contact. From dispersion corrected DFT methods, the energy difference between the T-shaped and stacked structures significantly increases, favoring the parallel ones in the series of benzene-naphthalene-anthracene, etc. Indeed, the parallel-displaced $(\pi-\pi$ interaction) conformation becomes the global minimum, already for the naphthalene case ${ }^{2}$. MP2 calculations of a set of PAHs shows $\pi$-stacking interactions of the parallel-displaced type again, sometimes with small in-plane rotation angles, with independence of the shape of the $\mathrm{PAH}$ analyzed as the lower energy candidates in the conformational space ${ }^{15}$. The most accurate theoretical estimation of the dimerization energy of naphthalene affords a value of $6.12 \mathrm{kcal} \mathrm{mol}^{-1}$ at the extrapolated $\operatorname{CCSD}(\mathrm{T}) /$ ha-cc-pVDZ level of theory ${ }^{16}$, with a face-toface interaction of rotated-shifted geometry. Dispersion corrected DFT methods also found a parallel shifted (not-rotated) geometry as a global minimum of close binding energy, more stable that the sigma- $\pi$ tilted T-shaped (or tilted $\mathrm{CH}-\pi$ ) by $0.69 \mathrm{kcal} \mathrm{mol}^{-1}{ }^{5}$ Unfortunately there are no specific experimental data with which the calculated dimer energies for naphthalene and anthracene could be compared, although gas phase excitation spectra of clusters consisting of naphthalene dimers with a number of Ar atoms seem to indicate the presence of T-shaped dimers ${ }^{17}$.

Our working scheme instead takes a heuristic approach for the study of these interactions. We focused our efforts in the structural information disclosed by the crystal structure of the most common families of benzenoid PAHs. From there, the fundamental dimer interaction was analyzed and the energy of that interaction was computationally evaluated. The role of electron correlation in the description of this interaction was evaluated at this point, showing that not only the dispersion terms, but also the charge-transfer properties are influenced by an adequate evaluation of the electron correlation. Almost without exception, the edge-toface (or $\mathrm{CH}-\pi$ ) type of interaction was found to account for the majority of the lattice energy $(\geq 50 \%)$, which is available from the experimental sublimation enthalpy of the arene series considered $^{18}$.

\section{Crystal Analysis}

Details from molecular association in the solid state can be regarded as the most reliable and fairly comprehensive set of experimental data available. Thus, members of increasing size of acenes, phenacenes and p-phenyls were carefully examined to identify the type of intermolecular interactions involved. In all cases, an inexorable structural motif was found to take a leading role in the solid state architecture: the herringbone pattern. This well-known array is formed by self-organization of the individual molecules in a repetitive edge-to-face fashion, conforming a plane that is the principal structural element of the ensemble. Four 
edge-to-face interactions surround each molecule approximately in the plane defined by the two shortest crystal cell axes, e.g., $a$ and $b$. In two of them the molecule is a $\pi$ component, and in the other two is a $\mathrm{CH}$ component of the interaction with the neighbor molecule. The final crystal is formed by piling up these herringbone planes through the direction of the longest crystal cell axis, e.g., c. This crystal structure is not only found in all the PAHs considered here that include the acene, phenacene and p-phenyl series, but it is a pervasive element found in most crystal structures of aromatic hydrocarbons. Figure 1 depicts the archetypal arrangement of arene molecules within the aforementioned ab plane of the crystal for a representative acene, phenacene and p-phenyl. The peculiarities of the interaction for each type of crystal are gathered in the Supporting information. In those cases in which more than one phase were available (e.g. anthracene, crysene), only the most stable phase under ambient conditions was analyzed. For benzene, the phase I was chosen (phase I is the first solid occurring either by increasing $\mathrm{P}$ or lowering $\mathrm{T}$ starting from liquid benzene at standard ambient conditions). Notice that in Figure 1, A represents a $\mathrm{D}_{2 h}$ or $\mathrm{C}_{2 h}$ molecule (acenes, p-phenyls and $[\mathrm{n}]$ phenacenes, $\mathrm{n}$ even) and $\mathrm{C}$ represents a $\mathrm{C}_{2 v}$ molecule ([n]phenacenes, $\mathrm{n}$ odd). The arrow represents the direction of the donor-acceptor interaction.

\section{Enthalpy of sublimation: experimental data}

In order to check the validity of the numerical methods used to describe bonding in PAH crystals, their sublimation enthalpies were employed. Experimental data for the sublimation enthalpy $\Delta H_{S}^{e}$ of eleven PAH (five acenes, three phenacenes and three phenyls) reported in current literature, are gathered in Table 1. As all experimental data were taken at room temperature whereas calculations were done at $0 \mathrm{~K}$, a temperature correction that resulted to be small and almost constant for all molecules (see below), was added to all experimental data. The monotonic increase of the sublimation enthalpy with the number of carbon atoms $N_{C}$ in each building molecule is obvious. A more quantitative illustration of this qualitative observation is attained by plotting the sublimation enthalpy versus $N_{C}$, as it is done in Fig. 2. Data apparently follow a straight line no matter the group to which the molecules belong (fittings are of course only valid for $N_{C} \geq 6$ ). This is corroborated by the goodness of the linear least squares fitting to all the data (correlation coefficient 0.992). We shall go back later to the experimental slope value of 6.72 in Fig 2, since this is a key piece of information in this work. This linear relationship between the sublimation enthalpy and the number of atoms in the molecule can be understood in the following terms. As explained in the crystal analysis section (see also Figure 1), from a structural point of view there are two main contributions to the lattice energy. The crystal builds up in the ab plane using the edge-to-face interaction, which is the one being studied theoretically in here. The thickness of this layer is, in a rough approximation, proportional to the full length of the molecule, so the larger the molecule, the stronger the edge-to-face interactions are. The molar binding energy within this layer (or intralayer contribution), is therefore expected to be proportional to $N_{C}$. On the other hand, in the c direction, the crystal grows by stacking ab layers, one above the other. This results in another contribution to the reticular energy due to interlayer fitting, now in the c direction (interlayer contribution). This weaker interaction is mainly of the intermolecular H-H type, occurring between layers. But more importantly, the molar 
binding energy in this direction is relatively constant regardless of the size of the molecule, therefore independent of $N_{C}$.

Fragment-based methods study crystals decomposing the total energy of an arrangement of interacting molecules in the crystal according to a many-body expansion, which includes 1-body, 2-body, 3-body terms, ... etc, and piece them together to obtain the energy of the system as a whole ${ }^{19}$. Having identified the $\mathrm{CH}-\pi$ dimers as the main interaction conforming these crystals, the potential role of trimers in the overall lattice energy was evaluated. For the naphthalene and anthracene cases, starting from a central molecule as the center of a trimer, we calculated the two possible lineal trimers made with the closest neighbors in the ab plane, and averaged them (e.g. as in Figure 1, center molecule in red, and the array left-centerright molecules as the first trimer, and top-center-down molecules as the second trimer). The differences in binding energy in the 3-body terms as compared to twice the binding energy of the 2-body terms are rather insignificant, well below the $\mathrm{kJ} / \mathrm{mol}(-0.12 \mathrm{~kJ} / \mathrm{mol}$ and $0.05 \mathrm{~kJ} / \mathrm{mol}$ for naphthalene and anthracene at the M06-2X-D3; $-0.44 \mathrm{~kJ} / \mathrm{mol}$ and $0.41 \mathrm{~kJ} / \mathrm{mol}$ for M06-2X, both with the def2-SPVD ${ }^{20}$ basis set).

In summary, intralayer interactions are proportional to the length of the molecule, which grows linearly with $N_{C}$, while interlayer interactions depend on the width (or the section) of the molecule, which is relatively constant and independent of $N_{C}$. This is due to the similar type of packing displayed by these series of molecules, and the overall similar shapes. The overall result is the linear relationship between the two magnitudes illustrated in Fig. 2.

\section{Methods}

\section{Temperature corrections applied to sublimation enthalpies}

The sublimation enthalpy of a solid at a temperature $T, \Delta H_{S}(T)$, is given by the general equation:

$$
\begin{aligned}
\Delta H_{S}(T) & =\Delta H_{S}(0)-\int_{0}^{T}\left[C_{P}^{s}(T)-C_{P}^{g}(T)\right] \mathrm{d} T \\
\Delta H_{S}(0) & =-E_{l a t}+\Delta E_{Z P E}
\end{aligned}
$$

where $C_{P}^{s}(T)$ and $C_{P}^{g}(T)$ are the heat capacities of the solid and gas phases, respectively. $E_{l a t}$ and $\Delta E_{Z P E}$ stand for the lattice and zero point energies, respectively. The magnitude of the heat capacity of the gas phase is generally smaller than that of the solid phase due to the contribution of phonons in the crystal, therefore sublimation enthalpies decrease as temperature increases. Customarily, sublimation enthalpies are referred to the standard temperature, $298.15 \mathrm{~K}^{21}$ although the experimental measurement is usually performed at a more suitable temperature $T_{m}$ defined in terms of mass-transport efficiency. Since this temperature depends on each particular compound, some linear corrections based on the solid and the gas phase heat capacities difference $C_{P}^{s}(T)-C_{P}^{g}(T)$ at the midpoint of the temperature range $T_{m}-298.15 \mathrm{~K}^{22}$ or in the absence of experimental data, by means of an estimated $C_{P}^{s}(298.15 K)$ by group additivity methods ${ }^{23}$ are usually applied. These approaches are valid if a narrow range of temperatures is involved but are not acceptable to estimate the sub- 
limation enthalpy corrections down to $0 \mathrm{~K}$, since heat capacities tend quickly to 0 as we approach this temperature overestimating corrections.

In order to obtain a reliable estimate of this correction, entire expressions of $C_{P}^{s}(T)$ and $C_{P}^{g}(T)$ will be inserted in eq. 1. For the solid phase this approach requires $C_{P}^{s}(T)$ measured from temperatures near the absolute zero to room temperature, which are available for a majority of the PAHs here considered. In cases in which several calorimetric studies were available, we used the criteria of higher quality of data based on method, number of measurements, purity of the sample, etc. as specified elsewhere ${ }^{24}$. For the gas phase heating, statistical thermodynamics provides a general and accurate scenario extendable to all PAHs. Let $Q(T)$ be the molecular partition function given by

$$
Q(T)=\Sigma_{i=1, . ., N_{v}} e^{-\frac{\epsilon_{i}}{k T}}
$$

where $\epsilon_{i}$ are the energies of the $N_{v}$ molecular vibrational modes (or vibrons) which can be extracted from the quantum mechanical calculations for the monomer. Then,

$$
\Delta H^{g}(T)-\Delta H^{g}(0)=\int_{0}^{T} C_{P}^{g}(T) \mathrm{d} T=\frac{R T^{2}}{Q(T)} \frac{\partial Q(T)}{\partial T}+R T .
$$

Table 2 gathers all experimental data needed for the estimation of the temperature correction to be added to the experimental data to bring them from room temperature (temperature at which measurements were carried out) to $0 \mathrm{~K}$ (temperature at which all calculations were done). As all calculated values are around $4 \mathrm{~kJ} / \mathrm{mol}$, the correction for those molecules for which there was not enough experimental information, was taken equal to that value.

Comparison of the experimental sublimation enthalpies $\Delta H_{S}(T)$ with the calculated lattice energies $E_{\text {lat }}$ requires assessing the vibrational contributions, not only thermal (as has been done in Table 2) but also zero-point energies of the solid and the gas phase, to evaluate their importance in the overall enthalpy according to the previous general expressions. In the high temperature limit, the Dulong-Petit law provides an estimate to these contributions, $\Delta H_{S}(T)=-E_{\text {lat }}+2 R T^{25,26}$, which reduces to $2 R T(4.96 \mathrm{~kJ} / \mathrm{mol})$ the applied correction. This correction is attributed to twice the phonon energy in the high temperature limit of the solid $(6 R T)$, once the thermal energy $(3 R T)$ and expansion work $(R T)$ of the gas phase are subtracted, and also circumvents the problem of calculating vibrational contributions involving solids (e.g. $\Delta E_{Z P E}$, which is neglected). This approach turned out to fit quite well for the molecular solids considered in this work, according to the experimental integrated heat capacities collected in Table 2, which are very close to this value (corrections are usually less than $1 \mathrm{~kJ} / \mathrm{mol}$ away from $2 R T$ ). A number of approaches that evaluate with more detail the computation of vibrational contributions to the sublimation enthalpy in some sets of crystal clusters (unit cells and supercells) have been the object of recent attention ${ }^{25}$. It is however not the purpose of this work to stretch the limits of the accuracy for any specific calculation, but to provide an overall view of the main structural motifs displayed in the crystal packing by these families of compounds, and the underlying causes that led to them. In this context, all the applied (thermal) and non-applied (zero-point) corrections are rather small terms that do not affect significantly the conclusions of this work (corrections are less than $3 \mathrm{~kJ} / \mathrm{mol}$ away from $2 R T$ for naphthalene and anthracene in the harmonic limit, as stated in reference ${ }^{25}$. 
Overall, seven out of eleven arenes are included (naphthalene, biphenyl, anthracene, phenanthrene, tetracene, p-terphenyl and p-quaterphenyl, see Supporting information) to which benzene has been added to deal with a larger set of compounds. As all corrections are around $4 \mathrm{~kJ} / \mathrm{mol}$, this value was used for the remaining arenes (chrysene, pentacene and picene). It is very close although more accurate than the generic correction of $2 R T$ derived from the Dulong-Petit molar heat capacity of a solid and an ideal gas, customarily used when no $C_{P}^{s}$ data of the solid state is available ${ }^{22}$.

\section{Quantum Chemistry calculations}

Quantum chemical calculations were done with the Gaussian09 version D.01 ${ }^{27}$, the GAMESS ${ }^{28}$, plus the NwChem ${ }^{29}$ for optimizations. The SAPT analysis was made with the Psi4 program $^{30}$. As graphical interfaces we employed several packages as xmgrace, Mercury ${ }^{31}$, Molden $^{32}$ and Gabedit ${ }^{33}$. Two alternative methods, namely, one within the Density Functional Theory (DFT) framework, M06-2X $\mathrm{X}^{34}$, that belongs to the family Hybrid MetaGGA Density Functionals, and the 2nd order perturbative method proposed by MollerPlesset (MP2). The pairwise dispersion energy has been complemented through the Grimme D3 correction (-D3) ${ }^{35}$, parametrized for the M06-2X functional. The best combination method/basis set, most compatible with our computing facilities, was chosen after an extensive study of small dimers (benzene and naphthalene, see Supporting information). The counterpoise correction $(\mathrm{CP})^{36}$ has been included in all calculations.

The goodness of the fitting of the experimental data for the heat of sublimation of the crystalline phases by means of a straight line clearly illustrates the linear relationship between heat of sublimation and number of carbons, or, equivalently, the average number of $\mathrm{CH}-\pi$ bonds, which justifies our heuristic approach: approximating the sublimation enthalpy by twice the total energy of the dimer $E_{d}$ minus that of the two monomers $E_{m_{i}}$, specifically,

$$
\Delta H_{S}^{t}=2\left(E_{d}-\sum_{i=1,2} E_{m_{i}}\right)
$$

The factor of 2 is justified by the fact that there are four edge-to-face interactions in the unit cell, and two molecules per cell. $E_{d}$ was calculated by taking the carbon coordinates from crystallographic data (see Supporting information) and optimizing those of hydrogens. This procedure is forced by the fact that only carbon coordinates can be accurately determined by X-ray crystallography, while hydrogen idealized coordinates are added by modeling. In addition the monomers total energy $E_{m_{i}}$ was calculated by taking the coordinates in the monomer of all atoms equal to those in the dimer.

\section{Inter- versus Intralayer interactions}

The structural arguments put forward in the Introduction under the heading "Experimental data", allows us to dissect the overall lattice energy in two smaller components easier to handle, on one hand the ab interlayer, a relatively constant contribution to the lattice energy, and on the other hand the ab intralayer, a linear contribution with $\mathrm{N}_{C}$ to the lattice energy. This approach has been tested in Table 3, for two small members (naphthalene and 
anthracene) by reexamining a more complete neighborhood of interactions surrounding a specific molecule in the network. Following the work of Sancho et al. ${ }^{37}$, a $2 \times 2 \times 2$ supercell was build, making the central molecule of the ensemble the origin $(0,0,0)$. From that perspective, an overall scenario of 38 interactions around the central molecule are accounted for, which considering the symmetry of the network becomes reduced to 12 different interactions of pairs of molecules to be calculated, all of them between the reference molecule located at $(0,0,0)$ and a second molecule of the 38 in the neighborhood. That second one is named in Table 3 using the fractional coordinates (Fract. coord.) system. We have extended the calculation of pairs within the supercell to the three methods considered in this work (M06-2X, M06-2X-D3 and MP2, using the def2-TZVPP basis set). As a general comment, from the overall calculated lattice energy (last row) it is easily seen that adding the dispersion term to DFT calculations provides a good estimate of the experimental $\Delta H_{s}$ corrected to $0 \mathrm{~K}$ for these crystals (100.5\% for naphthalene and $90.1 \%$ for anthracene, from M06-2X-D3). DFT alone underestimates, while MP2 overestimates the lattice energy in spite of the counterpoise correction. The first four rows in Table 3, correspond to the ab intralayer interactions (they can be recognized since the $c$ component of the fractional coordinates is zero). Among them, the first one, named $(\mathrm{a} / 2, \mathrm{~b} / 2,0)$, is of special significance since it corresponds to the $\mathrm{CH}-\pi$ bond considerate in this work (drawn in Figure 1). This structural element alone is by far the major energy contributor to the overall lattice energy, and increases from naphthalene $(37.6 / 77.28=48.7 \%)$ to anthracene $(52.23 / 95.16=54.9 \%)$, as expected (M06-2X-D3 data). Already for anthracene, the CH- $\pi$ bond accounts for more than $50 \%$ of the overall lattice energy, and it is expected to increase with $N_{C}$ in larger members, regardless of the method used. The next three rows correspond to the remaining ab intralayer contributions, also linearly dependent with $N_{C}$. However, the remaining 8 contributions (they can also be recognized since the $c$ component of the fractional coordinates is exactly $c$ ) represent the ab interlayer. From the overall ab interlayer sum, it is noted that these contributions are almost independent of $N_{C}: 21.72 \mathrm{~kJ} / \mathrm{mol}$ for naphthalene and $20.55 \mathrm{~kJ} / \mathrm{mol}$ for anthracene both obtained with M06-2X-D3 (the results obtained with other methods follow similar trends).

\section{Results and Discussion.}

\section{Sublimation Enthalpies: The importance of $\mathrm{CH}-\pi$ bond- ing}

Fig. 3 shows two configurations of PAH dimers commonly found in the literature: the parallel-displaced and the T-shaped (I and II, which correspond to two close energy minima found by freeing all atomic coordinates during the dimer calculation, respectively. In the anthracene case, a M06-2X-D3/def2-TZVPP calculation shows that the parallel-displaced structure is $14.88 \mathrm{~kJ} / \mathrm{mol}$ more stable than the T-shaped.). The third III corresponds to that used as input in the present process: carbon coordinates taken from crystallographic data and hydrogens allowed to relax in every dimer given the lack of experimental coordinates on light atoms, as stated before. This is the starting configuration for every calculation.

Sublimation energies of all arenes shown in Table 1 were calculated using three com- 
binations of methods/basis set (three methods, two DFT-based and one perturbative, and just one basis set) that were chosen after the extensive study of benzene and naphthalene discussed above. Although in benzene, for instance, several geometries of the dimers with very similar energies are known to exist (See e.g. ref. ${ }^{38-40}$ ) here we adopted the dimer extracted out from the crystal as explained before. Uncorrelated and correlated results for the sublimation enthalpy are reported in the Table 1 and Fig. 2. Uncorrelated values have a sign opposite to that of experimental data, indicating repulsion instead of attraction. In addition they vary only weakly with the number of carbons. An evident conclusion one can derive from these results is the many-body origin of $\mathrm{CH}-\pi$ bonding.

A valid interpretation of the straight lines in Fig. 2 turned out to be intimately related to the structural features of the crystals. From the general equation of the lines $\Delta H_{S}=$ $m N_{C}+C$, the meaning of the slope $m$ is of special relevance. $m$ is directly connected to the strength of all the intralayer interactions, which conform the aforementioned ab plane and grow linearly with $N_{C}$. For the experimental line, $m^{e}=6.72$, the intralayer contributions is composed of the sum of the four $\mathrm{CH}-\pi$ interactions surrounding a given molecule in the network (that we call $m_{4}$ contribution) as well as the remaining interactions that occur between that molecule and any other molecule at a farther distance, up to the infinite (called $m_{\infty}$ contribution), always within the $a b$ plane. In algebraic terms, $m^{e}=m_{4}+m_{\infty}$. It is therefore immediate to realize that the slope of the theoretical lines, $m^{t}=3.84$ (M06-2X), 5.03 (M06-2X-D3) and 7.19 (MP2) account only for $\mathrm{m} 4$ as calculated with each theoretical method. Also, it then follows that the intercept of the experimental line $C^{e}$ is the remaining interlayer contribution to the molar sublimation enthalpy, $\Delta H_{S}^{e}$, which is approximately a constant value for all the crystals. Further validation of this interpretative scheme brought us to a recent work devoted to the dispersion-corrected DFT calculation of the lattice energy of anthracene ${ }^{37}$. We have applied that methodology to the naphthalene and anthracene crystals in the previous section, corroborating thus successfully this scheme. For each studied method, the ratio of slopes $\frac{m_{4}^{t}}{m^{e}}$ gives us an idea of the importance of the $\mathrm{CH}-\pi$ interaction in the overall lattice energy, particularly in the limiting case of molecules containing a large number of carbons, i.e. $N_{C} \longrightarrow \infty$. This expression gives for the three methods/basis set combinations the following results: $\frac{m_{4}^{M 06-2 X}}{m^{e}}=57 \%, \frac{m_{4}^{M 06-2 X-D 3}}{m^{e}}=75 \%$ and $\frac{m_{4}^{M P 2}}{m^{e}}=$ $107 \%$. So, we can conclude from this analysis that the major contribution to the lattice energy in these series of molecules is due to the $\mathrm{CH}-\pi$ interaction, accounting, for the most accurate method (M06-2X-D3), some $\frac{3}{4}$ of the whole intralayer interaction, and that MP2 perturbative methods clearly overestimates the interaction, while dispersion uncorrected DFT seem to underestimate it. For the interlayer contribution $C^{M 06-2 X-D 3}$, the interaction can be estimated as ca. $21.1 \mathrm{~kJ} / \mathrm{mol}$ (see previous section and Table 3 containing the itemization of the different contributions to the interlayer interaction).

These numbers represent a reliable test of the general performance of the different methods/basis sets used in the computation of this type of weak intermolecular interactions, of particular significance since an experimental set of data is being taken as a benchmark. 


\section{The nature of the $\mathrm{CH}-\pi$ interaction}

The various components contributing to the interaction energy were obtained using symmetryadapted perturbation theory (SAPT), which has already been successfully used to study some pluri-molecular complexes of carbon. In the SAPT method, the uncorrelated Hamiltonian of the dimer is the sum of the Fock operators for the monomers and the perturbation Hamiltonian contains two terms: the intra- and inter-molecular correlation operator. The total interaction energy is obtained as a sum of perturbative corrections corresponding to electrostatic (elec), induction (ind) and dispersion (disp) contributions and their respective exchange (exch) counterparts. Up to second order (super-indexes 1 and 2 denote 1st and 2nd order terms):

$$
E_{\text {int }}^{S A P T}=E_{\text {elec }}^{1}+E_{\text {exch }}^{1+2}+E_{\text {ind }}^{2}+E_{\text {disp }}^{2},
$$

where $E_{\text {ind }}^{2}$ and $E_{\text {disp }}^{2}$ include the $E_{\text {exch-ind }}^{2}$ and $E_{\text {exch-disp }}^{2}$ terms, respectively. Table 4 shows the results for the four smaller acenes, obtained with a basis set sufficiently large (aug-ccpVDZ). Note that in order to compare $E_{\text {int }}^{S A P T}$ with the experimental and calculated heats of sublimation it has to be multiplied by 2 (see above). Once this is done, the results are 1-12\% smaller than those obtained with direct MP2 (see Table 1). The results show the importance of the contribution of dispersion to the stabilization studied, versus the other attractive terms, the electrostatic and the induction ones. In the Supporting information we show the results modifying the basis set functions and with two different optimized geometries. These results clearly indicate the weak dependence of the final sublimation enthalpy on the method/basis set used for optimizing hydrogen coordinates.

A pictorial way to illustrate the essential features of the $\mathrm{CH}-\pi$ bond is to calculate the changes in the local density of electronic charge due to bonding and interactions. However, in order to pick up the changes that bonding induces in the total electronic charge one has to choose the relevant magnitude. What we have done is to calculate the difference between the change in the electronic density induced by dimerization given by the correlated (c) solution minus that of the uncorrelated $(\mathrm{u})$ HF one. Specifically, we plot in Fig. 4 the following magnitude,

$$
\Delta \rho=\left(\rho_{d}^{c}-\rho_{m 1}^{c}-\rho_{m 2}^{c}\right)-\left(\rho_{d}^{u}-\rho_{m 1}^{u}-\rho_{m 2}^{u}\right)
$$

where subindexes $m$ and $d$ stand for monomer and dimer, respectively, while $u$ and $c$ denote uncorrelated and correlated charge densities. In this expression the possibility of two different monomers ( $m 1$ and $m 2$ ) as may actually be the case, has been incorporated. Fig. 4 depicts the result derived from Eq. 7 for anthracene and tetracene. The features that are expected to characterize a $\mathrm{CH}-\pi$ bond are cleanly seen in this Figure. A similar result is obtained with the MP2 method (see Supporting information). This is so despite the non-negligible differences between the sublimation enthalpies provided by the two methods.

Non covalent interaction can be readily visualized using the NCI plot tool ${ }^{41}$, which is implemented in various software packages. The NCI pictures generated using HF, M06-2X and MP2 (i.e. non-correlated and correlated methods) for some of the dimers considered in this work have been examined and turned out to be quite similar in appearance. Our density difference plot, however, reveals the specific effect in the electron density distribution that is directly attributed to correlation effects, complementing thus the set of visualization tools available for studying weak interactions. 


\section{Concluding Remarks}

This work was addressed to assess the relevance of $\mathrm{CH}-\pi$ bonding (edge-to-face interaction) in the crystal herringbone-like architecture of Polycyclic Aromatic Hydrocarbons. To this end, state-of-the-art quantum chemistry technology, has been applied to evaluate quantitatively the percentage of the experimental sublimation enthalpy that this structural element covers. In so doing we have followed a heuristic approach and evaluated up to what extent the formation energy of those edge-to-face dimers describes the experimental sublimation energy of crystalline PAH. We have taken the coordinates of the carbon atoms from the experimental crystalline data, optimizing those of the hydrogen atoms, for which no structural data is generally available (unless neutron diffraction was used to solve the crystal). Isolation of the relevant molecular dimers allows to incorporate some of the most sophisticated tools of quantum chemistry and get a reliable picture of the isolated bond.

We have then analyzed the experimental data for the sublimation energy of crystalline PAH of the series of acenes (five), phenacenes (three) and p-phenyls (three). We have shown that all experimental sublimation energies, no matter to which family the PAH belongs to, are linearly related to the number of carbons in the molecule. Aiming to justify this linear relationship, we have argued that intra-layer interactions are proportional to the length of the molecule, which grows linearly with $N_{C}$, while interlayer interactions depend on the width (or the section) of the molecule, which is relatively constant and independent of $N_{C}$. This is due to the similar type of packing displayed by these series of molecules, and the overall similar shapes.

After a rather comprehensive analysis of benzene and naphthalene in which up to thirty combinations of method/basis set were used, we have decided to use three methods (two DFT-based and one perturbative) and one basis set, to calculate the sublimation enthalpies of the just mentioned eleven arenes. When compared to the experimental sublimation energy, the results are conclusive: this sole interaction is the largest contribution to the lattice energy, and definitively dictates the crystal architecture in all the studied cases. This has been further assessed by means of SAPT, a method that allows to identify the different contributions to the total energy. In addition, the dramatic failure of mono-determinantal methods (UHF) unambiguously proves the many-body origin of the $\mathrm{CH}-\pi$ bonding. The results show that while perturbative methods over-estimate dispersion effects, DFT-based methods likely underestimate those effects, an scenario that can be improved with the introduction of dispersion correction terms.

Finally we have shown that a representative picture of the bond can be provided by displaying the differences in calculated electron density between correlated and non-correlated methods: the expected characteristics of $\mathrm{CH}-\pi$ bonding are in this way fully revealed.

\section{Acknowledgement}

Financial support by the spanish "Ministerio de Ciencia e Innovación MICINN" (grants CTQ2011-24165, FIS2012-33521 and FIS2012-35880) and the Universidad de Alicante is gratefully acknowledged. We also acknowledge support from the DGUI of the Comunidad de Madrid under the R\&D Program of activities MODELICO-CM/S2009ESP-1691. 


\section{Keywords}

Keywords:

CH- $\pi$ Bonding, Polycyclic Aromatic Hydrocarbon Crystals, Sublimation

Enthalpies 


\section{References}

[1] I. G. Kaplan, Intermolecular Interactions, Wiley, New York, 2005.

[2] A. J. Stone, The Theory of Intermolecular Forces, Oxford University Press, Oxford, 2013.

[3] R. Podeszwa, K. Szalewicz, Phys. Chem. Chem. Phys. 2008, 10, 2735-2746.

[4] A. J. Misquitta, R. Podeszwa, B. Jeziorski, K. Szalewicz, J. Chem. Phys. 2005, 123, 214103.

[5] M. Alonso, T. Woller, F. J. Martín-Martínez, J. Contreras-García, P. Geerlings, F. DeâǍËProft, Chem. Eur. J. 2014, 20, 4931-4941.

[6] K. E. Riley, P. Hobza, Acc. Chem. Res. 2013, 46, 927-936.

[7] T. Bučko, J. Hafner, S. Lebègue, J. G. Ángyán, J. Phys. Chem. A 2010, 114, 1181411824.

[8] The CBS (complete basis set) limit values are evaluated by extrapolation exploiting the fact that the basis set error in the electron correlation energy is proportional to $N^{-3}$ for the aug-cc-pVNZ (aVNZ) basis set.

[9] S. K. Min, E. C. Lee, H. M. Lee, D. Y. Kim, D. Kim, K. S. Kim, J. Comput. Chem. 2008, 29, 1208-1221.

[10] K. S. Kim, S. Karthikeyan, N. J. Singh, J. Chem. Theory Comput. 2011, 7, 3471-3477.

[11] M. Pitoñák, P. Neogrády, J. Řezáč, P. Jurečka, M. Urban, P. Hobza, J. Chem. Theory Comput. 2008, 4, 1829-1834.

[12] Y. Cho, W. J. Cho, I. S. Youn, G. Lee, N. J. Singh, K. S. Kim, Acc. Chem. Res. 2014, 47, 3321-3330.

[13] C. R. Martinez, B. L. Iverson, Chem. Sci. 2012, 3, 2191-2201.

[14] M. Schnell, U. Erlekam, P. R. Bunker, G. von Helden, J.-U. Grabow, G. Meijer, A. van der Avoird, Phys. Chem. Chem. Phys. 2013, 15, 10207-10223.

[15] E. Yurtsever, J. Phys. Chem. A 2009, 113, 924-930.

[16] T. Janowski, P. Pulay, J. Am. Chem. Soc. 2012, 134, 17520-17525.

[17] C. Gilliéron, N. Sharma, K. Nauta, T. W. Schmidt, J. Phys. Chem. A 2007, 111, 4211-4214.

[18] M. Nishio, CrystEngComm 2004, 6, 130-158.

[19] G. J. O. Beran, S. Wen, K. Nanda, Y. Huang, Y. Heit, Top. Curr. Chem. 2014, 345, 59-93. 
[20] F. Weigend, R. Ahlrichs, Phys. Chem. Chem. Phys. 2005, 7, 3297-3305.

[21] M. V. Roux, M. Temprado, J. S. Chickos, Y. Nagano, J. Phys. Chem. Ref. Data 2008, 3\%, 1855-1996.

[22] J. S. Chickos, W. E. Acree, J. Phys. Chem. Ref. Data 2002, 31, 537-698.

[23] J. S. Chickos, D. G. Hesse, J. F. Liebman, J Structural Chemistry 1993, 4, 261-269.

[24] E. S. Domalski, E. D. Hearing, J. Phys. Chem. Ref. Data 1996, 25, 1-525.

[25] A. Otero-de-la Roza, E. R. Johnson, J. Chem. Phys. 2012, 137, 054103.

[26] A. M. Reilly, A. Tkatchenko, J. Chem. Phys. 2013, 139, 024705.

[27] M. J. Frisch, G. W. Trucks, H. B. Schlegel, G. E. Scuseria, M. A. Robb, J. R. Cheeseman, G. Scalmani, V. Barone, B. Mennucci, G. A. Petersson, H. Nakatsuji, M. Caricato, X. Li, H. P. Hratchian, A. F. Izmaylov, J. Bloino, G. Zheng, J. L. Sonnenberg, M. Hada, M. Ehara, K. Toyota, R. Fukuda, J. Hasegawa, M. Ishida, T. Nakajima, Y. Honda, O. Kitao, H. Nakai, T. Vreven, J. A. Montgomery, Jr., J. E. Peralta, F. Ogliaro, M. Bearpark, J. J. Heyd, E. Brothers, K. N. Kudin, V. N. Staroverov, R. Kobayashi, J. Normand, K. Raghavachari, A. Rendell, J. C. Burant, S. S. Iyengar, J. Tomasi, M. Cossi, N. Rega, J. M. Millam, M. Klene, J. E. Knox, J. B. Cross, V. Bakken, C. Adamo, J. Jaramillo, R. Gomperts, R. E. Stratmann, O. Yazyev, A. J. Austin, R. Cammi, C. Pomelli, J. W. Ochterski, R. L. Martin, K. Morokuma, V. G. Zakrzewski, G. A. Voth, P. Salvador, J. J. Dannenberg, S. Dapprich, A. D. Daniels, O. Farkas, J. B. Foresman, J. V. Ortiz, J. Cioslowski, D. J. Fox, Gaussian 09 Revision D.01, Gaussian Inc. Wallingford CT 2009.

[28] M. W. Schmidt, K. K. Baldridge, J. A. Boatz, S. T. Elbert, M. S. Gordon, J. H. Jensen, S. Koseki, N. Matsunaga, K. A. Nguyen, S. J. Su, T. L. Windus, M. Dupuis, J. A. Montgomery, J.Comput.Chem. 1993, 14, 1347-1363.

[29] M. Valiev, E. J. Bylaska, N. Govind, K. Kowalski, T. P. Straatsma, H. J. J. V. Dam, D. Wang, J. Nieplocha, E. Apra, T. Windus, W. A. de Jong, Comput. Phys. Commun. 2010, 181, 1477 - 1489 .

[30] J. M. Turney, A. C. Simmonett, R. M. Parrish, E. G. Hohenstein, F. A. Evangelista, J. T. Fermann, B. J. Mintz, L. A. Burns, J. J. Wilke, M. L. Abrams, N. J. Russ, M. L. Leininger, C. L. Janssen, E. T. Seidl, W. D. Allen, H. F. Schaefer, R. A. King, E. F. Valeev, C. D. Sherrill, T. D. Crawford, WIREs Comput. Mol. Sci. 2012, 2, 556-565.

[31] C. F. Macrae, I. J. Bruno, J. A. Chisholm, P. R. Edgington, P. McCabe, E. Pidcock, L. Rodriguez-Monge, R. Taylor, J. van de Streek, P. A. Wood, J. Appl. Cryst. 2008, 41, 466-470.

[32] G. Schaftenaar, J. H. Noordik, J. Comput.-Aided Mol. Design 2000, 14, 123-134.

[33] A.-R. Allouche, J. Comput. Chem. 2011, 32, 174-182. 
[34] Y. Zhao, D. Truhlar, Theor. Chem. Acc. 2008, 120, 215-241.

[35] S. Grimme, J. Antony, S. Ehrlich, H. Krieg, J. Chem. Phys. 2010, 132, 154104.

[36] S. F. Boys, F. Bernardi, Mol. Phys. 1970, 19, 553-566.

[37] J. C. Sancho-García, J. Aragó, E. Ortí, Y. Olivier, J. Chem. Phys. 2013, 138, 204304.

[38] E. Yurtsever, J. Phys. Chem. A 2009, 113, 924-930.

[39] R. Podeszwa, B. M. Rice, K. Szalewicz, Phys. Rev. Lett. 2008, 101, 115503.

[40] S. Ehrlich, J. Moellmann, S. Grimme, Acc. Chem. Res. 2013, 46, 916-926.

[41] E. R. Johnson, S. Keinan, P. Mori-Sánchez, J. Contreras-García, A. J. Cohen, W. Yang, J. Am. Chem. Soc. 2010, 132, 6498-6506.

[42] Handbook of vapor pressures and heats of vaporization of hydrocarbons and related compounds, (Eds.: R. C. Wilhoit, B. J. Zwolinski), College Station : Thermodynamics Research Center, 1971.

[43] I. M. Alecu, J. Zheng, Y. Zhao, D. G. Truhlar, J. Chem. Theory Comput. 2010, 6, 2872-2887.

[44] J. Zheng, I. M. Alecu, B. J. Lynch, Y. Zhao, D. G. Truhlar, Database of Frequency Scale Factors for Electronic Model Chemistries, http://comp.chem.umn.edu/freqscale/version3b2.htm.

[45] G. D. Oliver, M. Eaton, H. M. Huffman, J. Am. Chem. Soc. 1948, 70, 1502-1505.

[46] J. P. McCullough, H. L. Finke, J. F. Messerly, S. S. Todd, T. C. Kincheloe, G. Waddington, J. Phys. Chem. 1957, 61, 1105-1116.

[47] P. Goursot, H. L. Girdhar, J. E. F. Westrum, Comptes rendus hebdomadaires des séances de l'Académie des Sciences Serie C 1968, 266, 949-950.

[48] P. Goursot, H. L. Girdhar, E. F. W. Jr., J. Phys. Chem. 1970, 74, 2538-2541.

[49] H. L. Finke, J. F. Messerly, S. H. Lee, A. G. Osborn, D. R. Douslin, J. Chem. Thermodyn. 1977, 9, $937-956$.

[50] K. Saito, T. Atake, H. Chihara, Bull. Chem. Soc. Japan 1988, 61, 679-688.

[51] K. Saito, T. Atake, H. Chihara, Bull. Chem. Soc. Japan 1988, 61, 2327-2336.

[52] J. S. Binkley, J. A. Pople, W. J. Hehre, J. Am. Chem. Soc. 1980, 102, 939-947.

[53] R. Krishnan, J. S. Binkley, R. Seeger, J. A. Pople, J. Chem. Phys. 1980, 72, 650.

[54] F. Jensen, J. Chem. Phys. 2001, 115, 9113-9125.

[55] F. Jensen, J. Chem. Phys. 2002, 116, 7372-7379. 
[56] F. Jensen, J. Chem. Theory Comput. 2008, 4, 719-727.

[57] J. Dunning, T. H., J. Chem. Phys. 1989, 90, 1007-1023.

[58] E. San-Fabián, F. Ingrosso, A. Lambert, M. I. Bernal-Uruchurtu, M. F. Ruiz-López, Chem. Phys. Lett. 2014, 601, $98-102$.

[59] E. G. Hohenstein, S. T. Chill, C. D. Sherrill, J. Chem. Theory Comput. 2008, 4, 19962000.

[60] M. Walker, A. J. A. Harvey, A. Sen, C. E. H. Dessent, J. Phys. Chem. A 2013, 117, 12590-12600.

[61] T. Janowski, P. Pulay, Theor. Chem. Acc. 2011, 130, 419-427. 


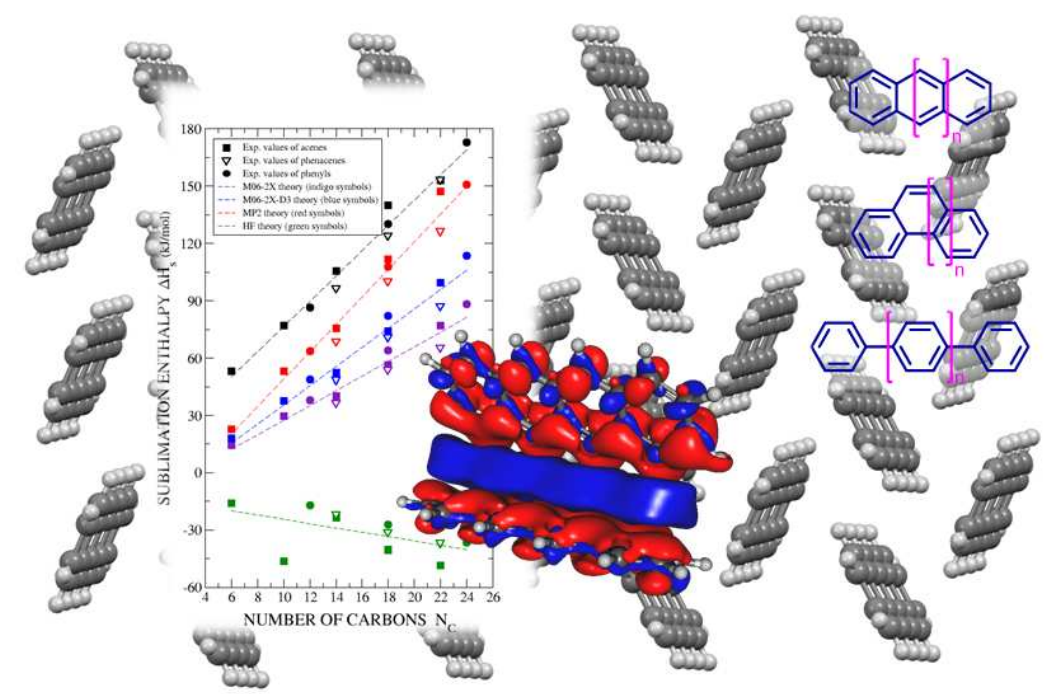

\section{Short text for the Table of Contents}

The experimental sublimation enthalpies of the acene, phenacene and p-phenyl series vs. molecular size reveals a general, linear behavior of these linear polycyclic aromatic hydrocarbons. The analysis of the cohesive forces in these compounds establishes the relevance of the $\mathrm{CH}$-pi bonding (edge-to-face interaction) in their crystal herringbone-like architecture, indicating that this sole interaction is the largest contribution to the lattice energy, dictating the crystal architecture in all cases. An illustrative picture of this elusive bond, entirely dependent on correlation effects and key to many molecular crystals of aromatic compounds, is provided by displaying the differences in calculated electron density between correlated and non-correlated methods. 



Figure 1: Upper: Terphenyl is the simplest case of a crystal network. All molecules in the cell are symmetrically equivalent (e.g. A). There is only one type of $\mathrm{CH}-\pi$ interaction of the type $\mathrm{A} \rightarrow \mathrm{A}$; all 4 bonds surrounding a molecule are equivalent. Middle; Tetracene. There are two symmetrically non-equivalent molecules in the cell (e.g. A and A). There are then two types of $\mathrm{CH}-\pi$ bonds, $\mathrm{A} \rightarrow \mathbf{A}$ and $\mathbf{A} \rightarrow \mathrm{A}$ (violet and pink wide arrows, respectively) and therefore two distinct pairs of equivalent bonds surrounding every molecule. Lower: Picene. All molecules in the cell are symmetrically equivalent (e.g. $\subset$ ). Due to its molecular point symmetry, there are two types of $\mathrm{CH}-\pi$ bonds, $\subset \rightarrow \subset$ and $\subset \rightarrow \supset$, accounting for the two possible edges of the molecule (violet and pink wide arrows, respectively) and therefore two distinct pairs of equivalent bonds surrounding every molecule. An arbitrary molecule was tinted in red for emphasizing in all cases. The ab plane (view shown) is defined by the two shorter crystallographic axes (in red and green), while the largest axis c (in blue) covers the full length of the molecule. 


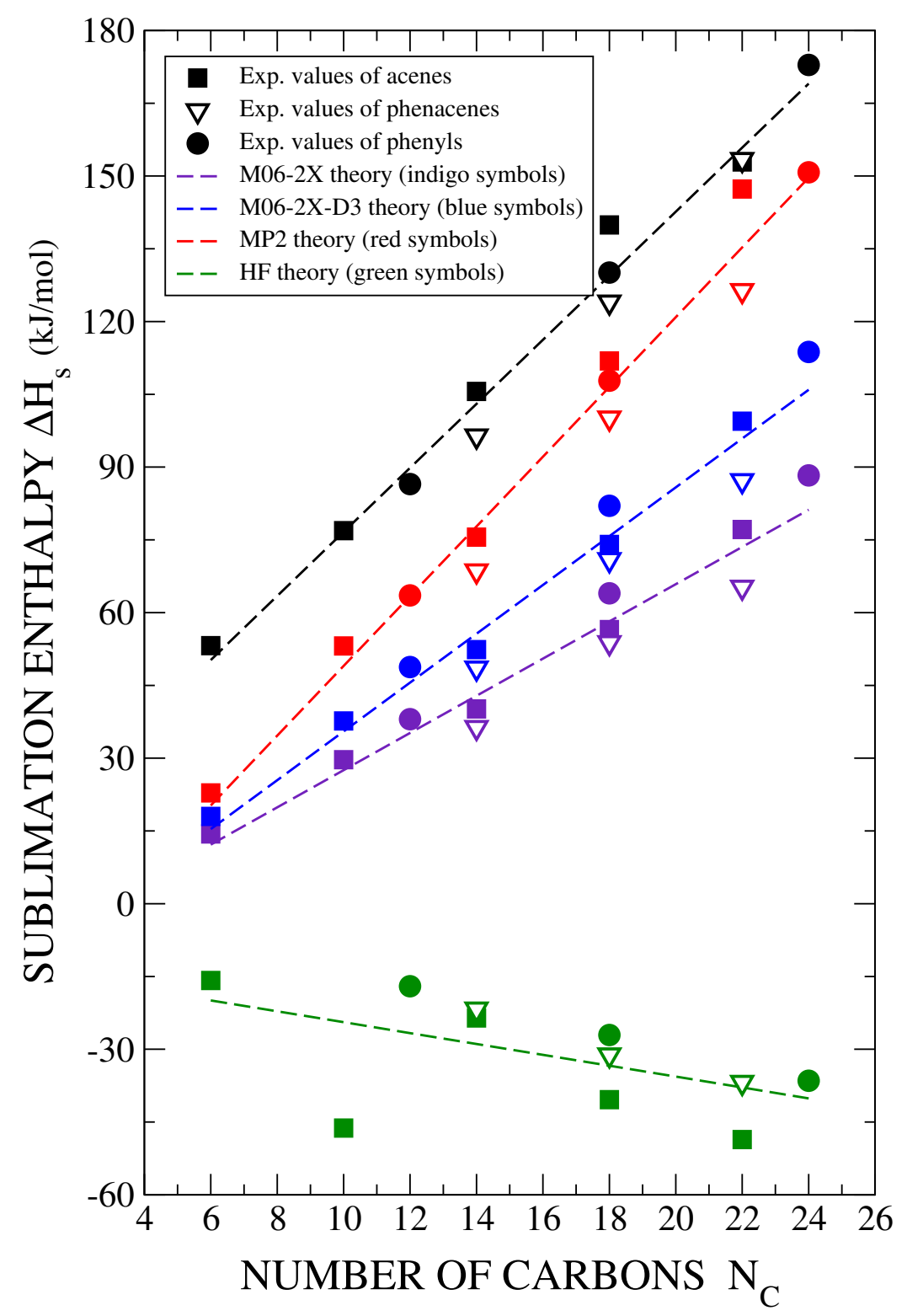

Figure 2: Heat of sublimation of the crystal phases of the acenes, phenacenes and phenyls gathered in Table 1. The experimental data admit a linear fitting as a function of the number of carbons no matter to which group they belong. Theoretical result obtained either with an uncorrelated approach or with a correlated method are also shown. The fact that uncorrelated results are in all cases negative, and almost independent of the number of carbons, is due to the failure of uncorrelated methods to describe bonding in these systems. The fitted straight lines are the following: $\Delta H_{S}^{e}=8.24+6.72 N_{C} \mathrm{~kJ} / \mathrm{mol} ; \Delta H_{S}^{M 06-2 X}=$ $-10.82+3.84 N_{C} \mathrm{~kJ} / \mathrm{mol} ; \Delta H_{S}^{M 06-2 X-D 3}=-14.78+5.03 N_{C} \mathrm{~kJ} / \mathrm{mol} ;$ and, $\Delta H_{S}^{M P 2}=-22.8$ $+7.19 N_{C} \mathrm{~kJ} / \mathrm{mol}$. All fittings valid for $N_{C} \geq 6$. 


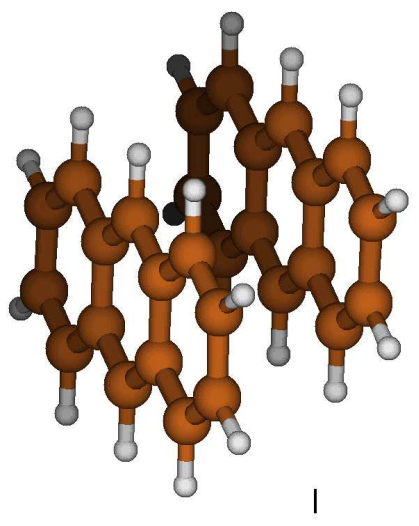

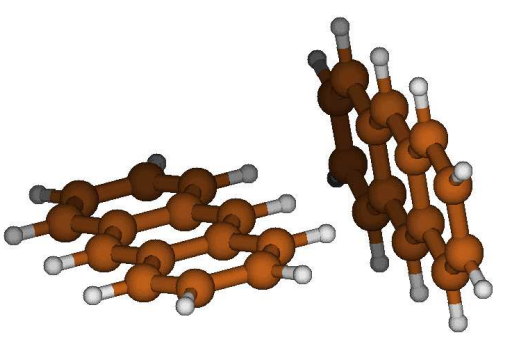

II

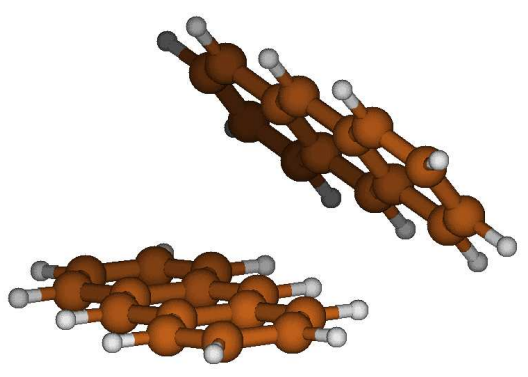

III

Figure 3: Depicts two standard configurations of PAH dimers (anthracene dimers in this figure) widely investigated in the literature, i.e., the parallel-displaced (I) and the tilted T-shaped (II), plus the one proposed in this work III, namely, the configuration in the crystalline counterpart of the molecule (see text).


Figure 4: Electronic charge related to bonding (see Eq. 7) in anthracene (left) and tetracene (right) dimers calculated with the combination M06-2X/def2-TZVPP (uncorrelated charge density was calculated with the same basis set). The geometry was determined by using the method proposed in this work, that is, carbons coordinates taken from crystallographic data and hydrogen coordinates optimized by means of the M06-2X/def2-SVPD combination. Blue colour indicates positive sign, i.e. excess electronic density. 
Table 1: Experimental data ${ }^{22}$ for the enthalpy of sublimation $\Delta H_{S}^{e}$ corrected to $\mathrm{T}=0 \mathrm{~K}$ of some acenes, phenacenes and p-phenyls (below uncorrected data in italics, ref. ${ }^{22}$ ), and calculated results $\Delta H_{S}^{t}$ of Eq. (5) (both in $\mathrm{kJ} / \mathrm{mol}$ ) obtained using M06-2X, M06-2X-D3 and MP2 methods with the basis set def2-TZVPP (see text). In parentheses, the percentage of the corrected experimental enthalpy of sublimation attained by the numerical results. The Counterpoise correction (CP) was also included.

\begin{tabular}{|c|c|c|c|c|c|c|}
\hline \multirow[t]{2}{*}{ Molecule } & \multirow[t]{2}{*}{ Formula } & \multirow[t]{2}{*}{$\overline{\Delta \Delta H_{S}^{e}(\mathrm{~T}=0 \mathrm{~K})}$} & \multicolumn{4}{|c|}{$\overline{\Delta \Delta H_{S}^{t}}$} \\
\hline & & & $\mathrm{HF}$ & M06-2X & M06-2X-D3 & MP2 \\
\hline \multirow[t]{2}{*}{ benzene } & $\mathrm{C}_{6} \mathrm{H}_{6}$ & 49.4 & -15.82 & 14.37 & 17.99 & 22.85 \\
\hline & & $45.2 \pm 0.2$ & - & $(29.0)$ & $(36.4)$ & $(46.3)$ \\
\hline \multirow[t]{2}{*}{ naphthalene } & $\mathrm{C}_{10} \mathrm{H}_{8}$ & 76.9 & -46.24 & 29.69 & 37.68 & 53.14 \\
\hline & & $72.6 \pm 0.3$ & - & $(38.6)$ & $(49.0)$ & $(69.1)$ \\
\hline \multirow[t]{2}{*}{ anthracene } & $\mathrm{C}_{14} \mathrm{H}_{10}$ & 105.6 & -23.55 & 40.15 & 52.38 & 75.57 \\
\hline & & $101.9 \pm 1.3$ & - & $(38.0)$ & $(49.6)$ & $(71.6)$ \\
\hline \multirow[t]{2}{*}{ tetracene } & $\mathrm{C}_{18} \mathrm{H}_{12}$ & 139.9 & -40.38 & 56.50 & 74.05 & 111.89 \\
\hline & & $135.9 \pm 5$ & - & $(40.4)$ & $(52.9)$ & $(80.0)$ \\
\hline \multirow[t]{2}{*}{ pentacene } & $\mathrm{C}_{22} \mathrm{H}_{14}$ & 152.9 & -48.61 & 77.13 & 99.46 & 147.32 \\
\hline & & $148.9 \pm 4.2$ & - & $(50.4)$ & $(65.0)$ & $(96.4)$ \\
\hline \multirow[t]{2}{*}{ 3_Phenacene } & $\mathrm{C}_{14} \mathrm{H}_{10}$ & 96.4 & -21.72 & 36.32 & 48.57 & 68.59 \\
\hline & & $92.1 \pm 0.6$ & - & $(37.7)$ & $(50.4)$ & $(71.2)$ \\
\hline \multirow[t]{2}{*}{ 4_phenacene } & $\mathrm{C}_{18} \mathrm{H}_{12}$ & 124 & -31.17 & 53.80 & 70.91 & 100.10 \\
\hline & & $120\left[^{21}\right]$ & - & $(43.1)$ & $(56.9)$ & $(80.7)$ \\
\hline \multirow[t]{2}{*}{ 5_Phenacene } & $\mathrm{C}_{22} \mathrm{H}_{14}$ & 153.4 & -36.84 & 65.24 & 87.19 & 126.37 \\
\hline & & $149.4 \pm 2.9$ & - & $(42.5)$ & $(56.8)$ & $(82.4)$ \\
\hline \multirow[t]{2}{*}{ Biphenyl } & $\mathrm{C}_{12} \mathrm{H}_{10}$ & 86.5 & -17.01 & 38.04 & 48.78 & 63.54 \\
\hline & & $82.1 \pm 2.1$ & - & $(44.0)$ & $(56.4)$ & $(73.4)$ \\
\hline \multirow[t]{2}{*}{ p-Terphenyl } & $\mathrm{C}_{18} \mathrm{H}_{14}$ & 130.1 & -27.06 & 63.98 & 82.02 & 107.82 \\
\hline & & $125.6 \pm 1.6$ & - & $(49.2)$ & $(63.0)$ & $(82.9)$ \\
\hline \multirow[t]{2}{*}{ p-Quaterphenyl } & $\mathrm{C}_{24} \mathrm{H}_{18}$ & 172.9 & -36.50 & 88.28 & 113.73 & 150.77 \\
\hline & & $168.4 \pm 1.6$ & - & $(51.1)$ & $(65.8)$ & $(87.2)$ \\
\hline
\end{tabular}


Table 2: Corrections to the calculated sublimation enthalpies $\Delta H_{S}(0 K)$ (in $\mathrm{kJ} / \mathrm{mol}$ ), from $\mathrm{T}=298.15 \mathrm{~K}$ to $0 \mathrm{~K}$, except for benzene, liquid at room temperature, the sublimation enthalpy of which is given at $269 \mathrm{~K}^{21,42}$ and corrections are from $\mathrm{T}=269 \mathrm{~K}$ to $0 \mathrm{~K}$. Column $3: A 1$, $\Delta H_{s}(R T)(\mathrm{kJ} / \mathrm{mol})$, Ref. ${ }^{21}$. Column 4: $A 2=\Delta H^{s}(0 K)-\Delta H^{s}(R T)(\mathrm{kJ} / \mathrm{mol})$, experimental integrated heat capacities of the solid. Column 5: $A 3$, experimental range of temperatures $(\mathrm{K})$ in $A 2$. Column 6: $A 4=\Delta H^{g}(0 K)-\Delta H^{g}(R T)(\mathrm{kJ} / \mathrm{mol})$, integrated Heat Capacity in the gas phase, including the translational $(3 / 2 \mathrm{RT}=3.718 \mathrm{~kJ} / \mathrm{mol})$, rotational $(3 / 2 \mathrm{RT}=$ $3.718 \mathrm{~kJ} / \mathrm{mol}$ ) and vibrational contributions from M06-2X/def2-TZVPP frequencies, as well as the expansion work $(\mathrm{RT}=2.479 \mathrm{~kJ} / \mathrm{mol})$. The vibrational frequencies were scaled by $0.983^{43,44}$. Column 7: $N_{v}$ number of normal vibrational modes followed by the symmetry point group of the molecule in the gas phase. Column 8: correction to $\Delta H_{S}(0 K)$ (in kJ/mol).

\begin{tabular}{lc|c|c|c|c|c|c}
\hline \hline arene & Formula & $A 1$ & $A 2$ & $A 3$ & $A 4$ & $N_{v}$ & correction \\
\hline benzene & $\mathrm{C}_{6} \mathrm{H}_{6}$ & 45.2 & $16.38^{45}$ & $12-341$ & 12.161 & $30 D_{6 h}$ & 4.22 \\
naphthalene & $\mathrm{C}_{10} \mathrm{H}_{8}$ & 72.6 & $24.79^{46}$ & $10-370$ & 20.518 & $48 D_{2 h}$ & 4.27 \\
anthracene & $\mathrm{C}_{14} \mathrm{H}_{10}$ & 101.9 & $31.24^{47,48}$ & $5-500$ & 27.523 & $66 D_{2 h}$ & 3.72 \\
tetracene & $\mathrm{C}_{18} \mathrm{H}_{12}$ & - & - & - & - & - & 4.0 \\
pentacene & $\mathrm{C}_{22} \mathrm{H}_{14}$ & - & - & - & - & - & 4.0 \\
\hline phenanthrene & $\mathrm{C}_{14} \mathrm{H}_{10}$ & 92.1 & $31.77^{49}$ & $10-420$ & 27.498 & $66 C_{2 v}$ & 4.27 \\
crisene & $\mathrm{C}_{18} \mathrm{H}_{12}$ & - & - & - & - & - & 4.0 \\
picene & $\mathrm{C}_{22} \mathrm{H}_{14}$ & - & - & - & - & - & 4.0 \\
\hline biphenyl & $\mathrm{C}_{12} \mathrm{H}_{10}$ & 82.1 & $30.45^{50}$ & $3-300$ & 26.003 & $60 C_{2}$ & 4.45 \\
p-terphenyl & $\mathrm{C}_{18} \mathrm{H}_{14}$ & 125.6 & $42.85^{51}$ & $5-300$ & 38.324 & $90 C_{2 h}$ & 4.53 \\
p-quaterphenyl & $\mathrm{C}_{24} \mathrm{H}_{18}$ & 172.5 & 55.36 & $5-300$ & 50.887 & $120 D_{2}$ & 4.47 \\
\hline \hline
\end{tabular}


Table 3: Interaction energies for the dimers of the crystalline structure of naphthalene and anthracene as taken from a 2x2x2 supercell (see text), at M06-2X, M06-2X-D3 and MP2 with the def2-TZVPP basis set. All in $\mathrm{kJ} / \mathrm{mol}$.

\begin{tabular}{|c|c|c|c|c|c|c|}
\hline \multirow[b]{2}{*}{ Fract. coord. } & \multicolumn{3}{|c|}{ Naphthalene } & \multicolumn{3}{|c|}{ Anthracene } \\
\hline & M06-2X & M06-2X-D3 & MP2 & M06-2X & M06-2X-D3 & MP2 \\
\hline \multicolumn{7}{|l|}{ Intralayer } \\
\hline$(\mathrm{a} / 2, \mathrm{~b} / 2,0)$ & 29.61 & 37.60 & 53.14 & 40.00 & 52.23 & 75.55 \\
\hline$(\mathrm{a}, 0,0)$ & -0.22 & 0.92 & 1.30 & -0.22 & 0.98 & 1.50 \\
\hline$(0, b, 0)$ & 11.76 & 15.13 & 17.24 & 14.43 & 19.51 & 25.53 \\
\hline \multirow[t]{2}{*}{$(a, b, 0)$} & 0.75 & 1.91 & 2.18 & 0.67 & 1.89 & 2.36 \\
\hline & 41.90 & 55.56 & 73.87 & 54.87 & 74.61 & 104.94 \\
\hline \multicolumn{7}{|l|}{ Interlayer } \\
\hline$(\mathrm{a} / 2, \mathrm{~b} / 2, \mathrm{c})$ & 5.83 & 10.10 & 14.73 & 8.43 & 13.65 & 18.12 \\
\hline$(-\mathrm{a} / 2, \mathrm{~b} / 2, \mathrm{c})$ & 0.27 & 0.81 & 1.00 & 0.13 & 0.49 & 0.69 \\
\hline$(0,0, c)$ & 2.71 & 4.41 & 6.37 & 1.49 & 3.06 & 5.69 \\
\hline$(\mathrm{a}, 0, \mathrm{c})$ & 1.13 & 3.02 & 3.47 & 0.71 & 2.21 & 2.63 \\
\hline$(-a, 0, c)$ & 0.05 & 0.09 & 0.11 & 0.04 & 0.07 & 0.08 \\
\hline$(0, b, c)$ & 0.54 & 1.54 & 1.78 & 0.30 & 1.02 & 5.26 \\
\hline$(a, b, c)$ & 0.34 & 1.66 & 1.93 & 0.02 & 0.99 & 1.41 \\
\hline \multirow[t]{2}{*}{$(-a, b, c)$} & 0.05 & 0.09 & 0.12 & 0.03 & 0.06 & 0.10 \\
\hline & 10.93 & 21.72 & 29.52 & 11.15 & 20.55 & 30.04 \\
\hline Lattice energy & 52.82 & 77.28 & 103.39 & 66.03 & 95.16 & 138.92 \\
\hline
\end{tabular}

Table 4: Interaction energies (in $\mathrm{kJ} / \mathrm{mol}$ ) for acenes dimers, calculated with the SAPT2 method and the aug-cc-pVDZ basis set. Deformation energy is not taken into account (i.e., the monomers geometry was assumed to be that in the dimers). The exchange term contains first and second order corrections (see text).

\begin{tabular}{lccccc}
\hline \hline System & $E_{\text {elec }}^{1}$ & $E_{\text {exch }}^{1+2}$ & $E_{\text {ind }}^{2}$ & $E_{\text {disp }}^{2}$ & $E_{\text {int }}^{S A P T}$ \\
\hline benzene & -9.165 & 22.789 & -1.202 & -23.668 & -11.246 \\
naphthalene & -22.311 & 54.621 & -2.412 & -53.516 & -23.618 \\
anthracene & -14.712 & 33.494 & -2.232 & -53.361 & -36.802 \\
tetracene & -22.504 & 53.340 & -3.483 & -80.244 & -52.888 \\
\hline \hline
\end{tabular}




\section{Supporting Information}

\section{Contents}

Some crystallographic data of the organic crystals investigated in this work . . .

Choosing a method/basis set combination: sublimation enthalpies of benzene and naphthalene . . . . . . . . . . . . . . . . . . 3

Complementary calculations . . . . . . . . . . . . . . . . . . . 4

\section{Some crystallographic data of the organic crystals inves- tigated in this work}

- Benzene Pbca: $\mathrm{C}_{6} \mathrm{H}_{6}, \mathrm{Z}=4$, a 7.243(3), b 9.31(2), c 6.756(3), $\alpha$ 90.00, $\beta$ 90.00, $\gamma$ 90.00. $\mathrm{V}=455.6(1) . \mathrm{d}=1.136 \mathrm{~g} / \mathrm{cm}^{3}$ at RT. All molecules are symmetrically equivalent (e.g. A). Only one type of $\mathrm{CH}-\pi$ interaction of the type $\mathrm{A} \rightarrow \mathrm{A}$ (all 4 bonds surrounding a molecule are equivalent). At 296(2) K, 0.3 GPa, Ref. A. Budzianowski, A. Katrusiak, Acta Crystallogr. (2006), 62, 94-101.

- Naphthalene P21/a: $\mathrm{C}_{10} \mathrm{H}_{8}, \mathrm{Z}=2$, a 7.6778(17), b 5.7210(10), c 8.395(3), $\alpha$ 90.00, $\beta$ 124.55(2), $\gamma$ 90.00. $\mathrm{V}=303.712 . \mathrm{d}=1.40 \mathrm{~g} / \mathrm{cm}^{3}$ at RT. Both molecules are symmetrically equivalent (e.g. A). There is only one type of $\mathrm{CH}-\pi$ interaction of the type $\mathrm{A} \rightarrow \mathrm{A}$ (all 4 bonds surrounding a molecule are equivalent). Ref. F. P. A. Fabbiani, D. R. Allan, S. Parsons, C. R. Pulham, Acta Crystallogr. (2006), B62, 826-842.

- Anthracene P21/a: $\mathrm{C}_{14} \mathrm{H}_{10}, \mathrm{Z}=2$, a 8.5526(12), b 6.0158(11), c 11.1720(16), $\alpha$ 90.00, $\beta$ 124.596(15), $\gamma$ 90.00. $\mathrm{V}=473.168 . \mathrm{d}=1.25 \mathrm{~g} / \mathrm{cm}^{3}$. at RT. Both molecules are symmetrically equivalent (e.g. A). There is only one type of $\mathrm{CH}-\pi$ of the type $\mathrm{A} \rightarrow \mathrm{A}$ (all 4 bonds surrounding a molecule are equivalent). Ref. C. Pratt Brock, J. D. Dunitz Acta Crystallogr. (1990), B46, 795-806.

- Tetracene P-1 ( $\mathrm{n}=2): \mathrm{C}_{18} \mathrm{H}_{12}, \mathrm{Z}=2$, a $6.0565(9)$, b 7.8376(11), c 13.0104(18), $\alpha$ $77.127(2), \beta$ 72.118(2), $\gamma$ 85.792(2). $\mathrm{V}=572.968 . \mathrm{D}=1.32 \mathrm{~g} / \mathrm{cm}^{3}$ at $175.15 \mathrm{~K}$. There are two symmetrically unequivalent molecules in the cell (e.g. A and A). Therefore there are two types of $\mathrm{CH}-\pi$ bonds, $\mathrm{A} \rightarrow \mathbf{A}$ and $\mathbf{A} \rightarrow \mathrm{A}$ (i.e., 2 distinct pairs of equivalent bonds surrounding a molecule). Both interactions have been calculated and averaged. Ref. D. Holmes, S. Kumaraswamy, A. J. Matzger, K. P. C. Vollhardt, Chem. Eur. J. (1999), 5, 3399-3412.

- Pentacene P-1 ( $\mathrm{n}=2): \mathrm{C}_{22} \mathrm{H}_{14}, \mathrm{Z}=2$, a $6.265(2)$, b 7.786(2), c 14.511(4), $\alpha$ 76.65(2), $\beta$ $87.50(2), \gamma 84.61(2) . \mathrm{V}=685.488 . \mathrm{D}=1.35 \mathrm{~g} / \mathrm{cm}^{3}$ at RT. There are two symmetrically unequivalent molecules in the cell (e.g. A and A). Therefore, there are two types of CH$\pi$ bonds (i.e., 2 distinct pairs of equivalent bonds surrounding a molecule), $\mathrm{A} \rightarrow \mathbf{A}$ and $\mathbf{A} \rightarrow$ A. Both interactions have been calculated and averaged. Ref. T. Siegrist, C. Kloc, J. H. Schon, B. Batlogg, R. C. Haddon, S. Berg, G. A. Thomas, Angew.Chem.,Int.Ed. (2001), 40, 1732-1736 (this phase has a denser packing that other polymorphs at RT). 
- Phenanthrene P21 ( $\mathrm{n}=4): \mathrm{C}_{14} \mathrm{H}_{10}, \mathrm{Z}=2$, a 8.441(2), b 6.140(1), c 9.438(1), $\alpha$ 90.00, $\beta$ 97.96(1), $\gamma$ 90.00. $\mathrm{V}=484.437$. Crystal $\mathrm{d}=1.22 \mathrm{~g} / \mathrm{cm}^{3}$ at RT. Both molecules are symmetrically equivalent (e.g. $\subset$ ). Since it's an arched-shaped molecule, there are two types of CH- $\pi$ bonds, of the type $\subset \rightarrow \subset$ and $\subset \rightarrow \supset$ (i.e., 2 distinct pairs of equivalent bonds surrounding a molecule). Both interactions have been calculated and averaged. Ref. V. Petricek, I. Cisarova, L. Hummel, J. Kroupa, B. Brezina, Acta Crystallogr. (1990), B46, 830-832.

- Chrysene $\mathrm{C} 2 / \mathrm{c}(\mathrm{n}=15): \mathrm{C}_{18} \mathrm{H}_{12}, \mathrm{Z}=4$, a 22.803(5), b 6.216(1), c 8.348(2), $\alpha$ 90.00, $\beta$ $96.49(3), \gamma 90.00 . \mathrm{V}=1175.69 . \mathrm{d}=1.29 \mathrm{~g} / \mathrm{cm}^{3}$ at RT. All molecules are symmetrically equivalent (e.g. A). There is only one type of $\mathrm{CH}-\pi$ interactions of the type $\mathrm{A} \rightarrow \mathrm{A}$ (all 4 bonds surrounding a molecule are equivalent). Ref. T. M. Krygowski, A. Ciesielski, B. Swirska, P. Leszczynski, Pol. J. Chem. (1994), 68, 2097-2107.

- Picene P21 ( $\mathrm{n}=4): \mathrm{C}_{14} \mathrm{H}_{10}, \mathrm{Z}=2$, a 8.480(2), b 6.154(2), c 13.515(3), $\alpha$ 90.00, $\beta$ 90.46(1), $\gamma$ 90.00. $\mathrm{V}=705.27$. Crystal $\mathrm{d}=1.31 \mathrm{~g} / \mathrm{cm}^{3}$ at RT. Both molecules symmetrically equivalent (e.g. $\subset$ ). Since it's an arched-shaped molecule, there are two types of $\mathrm{CH}-\pi$ bonds, of the type $\subset \rightarrow \subset$ and $\subset \rightarrow \supset$ (i.e., 2 distinct pairs of equivalent bonds surrounding a molecule). Both interactions have been calculated and averaged. Ref. A. De, R. Ghosh, S. Roychowdhury, P. Roychowdhury, Acta Crystallogr. (1985), C41, 907-909.

- Biphenyl P21/a: $\mathrm{C}_{12} \mathrm{H}_{10}, \mathrm{Z}=2$, a 8.120, b 5.640, c 9.470, $\alpha 90.00 \beta$ 95.40, $\gamma$ 90.00. $\mathrm{V}=$ 431.771. $\mathrm{d}=1.18 \mathrm{~g} / \mathrm{cm}^{3}$ at RT. Both molecules are symmetrically equivalent (e.g. A). There is only one type of $\mathrm{CH}-\pi$ interaction of the type $\mathrm{A} \rightarrow \mathrm{A}$ (all 4 bonds surrounding a molecule are equivalent). Ref. J. Trotter Acta Cryst. (1961). 14, 1135-1140.

- p-Terphenyl P21/a: $\mathrm{C}_{18} \mathrm{H}_{14}, \mathrm{Z}=2$. a 8.119(5), b 5.615(3), c 13.618(8), $\alpha$ 90.00, $\beta$ $92.07(3), \gamma 90.00 . \mathrm{V}=620.415 . \mathrm{d}=1.23 \mathrm{~g} / \mathrm{cm}^{3}$ at RT. Both molecules are symmetrically equivalent (e.g. A). There is then only one type of $\mathrm{CH}-\pi$ interaction of the type $\mathrm{A} \rightarrow \mathrm{A}$ (all 4 bonds surrounding a molecule are equivalent). Ref. J. L. Baudour, H. Cailleau, W. B. Yelon, Acta Crystallogr. (1977), B33, 1773-1780.

- p-Quaterphenyl P21/a: $\mathrm{C}_{24} \mathrm{H}_{18}, \mathrm{Z}=2$. a 8.110(6), b 5.610(4), c 17.91(1), $\alpha$ 90.00, $\beta$ $95.80(6), \gamma 90.00 . \mathrm{V}=810.682 . \mathrm{d}=1.25 \mathrm{~g} / \mathrm{cm}^{3}$ at RT. Both molecules are symmetrically equivalent (e.g. A). There is then only one type of $\mathrm{CH}-\pi$ interaction of the type $\mathrm{A} \rightarrow \mathrm{A}$ (all 4 bonds surrounding a molecule are equivalent). Ref. Y. Delugeard, J. Desuche, J. L. Baudour, Acta Crystallogr. (1976), B32, 702-705. 


\section{Choosing a method/basis set combination: sublimation enthalpies of benzene and naphthalene}

In order to choose a method/basis set compatible with our computing facilities, we have started using a large set of basis functions for small dimers (benzene and naphthalene) and two alternative methods. One within the Density Functional Theory (DFT) framework, M06-2X ${ }^{34}$, that belongs to the family Hybrid Meta-GGA Density Functionals, and the 2nd order perturbative method proposed by Moller-Plesset (MP2). The counterpoise correction $(\mathrm{CP})^{36}$ has been considered in all calculations.

Calculations were performed using a variety of basis sets. Minimal and extended basis sets, such as the more simple $3-21 \mathrm{G}^{52}$ and the fully polarized $6-311 \mathrm{G}^{* * 53}$ basis of Pople et al.. The double-Zeta, triple-Zeta and polarized double-Zeta basis sets optimized for DFT methods by Jensen, pc-1, pc- $2^{54,55}$, and the aug-pc- $1^{56}$. The Dunning's correlation-consistent basis set cc-pVDZ and aug-cc-pVDZ ${ }^{57}$. Finally, the Ahlrichs's split valence def2-SVPD, as well as the triple zeta valence def2-TZVPP ${ }^{20}$.

The goodness of the fitting of the experimental data for the heat of sublimation of the crystalline phases by means of a straight line clearly illustrates the linear relationship between heat of sublimation and number of carbons, or, equivalently, the average number of $\mathrm{CH}-\pi$ bonds, which justifies our heuristic approach: approximating the sublimation enthalpy by twice the total energy of the dimer $E_{d}$ minus that of the two monomers $E_{m_{i}}$, specifically,

$$
\Delta H_{S}^{t}=2\left(E_{d}-\sum_{i}^{2} E_{m_{i}}\right)
$$

Table S1 and Fig. S1 show the results for the sublimation enthalpies obtained with all basis sets here considered and the DFT M06-2X and the perturbative MP2 methods. The number of basis functions used to perform the calculations for the dimer are also shown as it is the only quantitative parameter that can be used to quantify the goodness of a basis set. Results derived from the perturbative method do vary steeply for small basis sets. However, they saturate for sets containing more than 400 functions. This behaviour is common to both benzene and naphthalene and is rather encouraging as, using for instance the basis set def2-TZVPP, we might be rather sure to be within the convergence region. DFT methods, as expected, are less sensitive to the quality of the basis sets. However, although they decrease very slowly with the number of functions in the basis set, they do not seem to saturate within the range covered by our results. It is, nonetheless, expected that saturation will show up sooner or later. These results convinced us to adopt the def2-TZVPP basis set for the main calculations presented hereafter. Some calculations will also be carried out with less extensive bases. In particular, in choosing the methods to carry out most calculations (see below) the def2-SVPD basis set was used, as the def2-TZVPP is too large for methods such as MP4 (4th order perturbation theory) or all CC (Coupled Cluster) methods.

Once chosen a basis set (def2-TZVPP), further calculations of the sublimation enthalpy of benzene and naphthalene, were carried out aiming to find out an adequate method for our purposes. In particular, besides the two methods mentioned and used above (M06-2X and MP2), calculations were carried out with the standard non-interacting method, i.e., the so-called Hartree-Fock (HF) method, perturbative methods incorporating corrections of 3rd 
order (MP3) and fourth order (MP4), the latter including either double and quadruple excitations (MP4(DQ)), single, double and quadruple excitations (MP4(SDQ)) or all possible excitations up to fourth order (MP4(SDTQ). Finally, Coupled Clusters (CC) methods considered to be the state-of-the-art in quantum chemistry including either single and double excitations (CCSD) or, in addition, an estimation of triple excitations (CCSD $(\mathrm{T})$ ). Extrapolated $\operatorname{CCSD}(\mathrm{T})$ has also been considered $(\operatorname{CCSD}(\mathrm{T})$ corrected for the residual basis set error that was calculated as the difference between results obtained with MP2/def2-SVPD and MP2/def2-TZVPP combinations). Although the method DFT M06-2X used above has been shown to be more powerful than other functionals in describing intermolecular interactions ${ }^{58}$ , and was configured to attain, among other things, a proper description of intermolecular energies, including, at least partially, dispersion effects ${ }^{59,60}$, it has been recently pointed out that the pairwise dispersion energy has to be concomitantly added through the Grimme D3 correction (-D3) ${ }^{35}$, parametrized for this functional.

Table S2 shows the sublimation enthalpies $\Delta H_{S}^{t}$ of benzene and naphthalene calculated with eleven methods and the def2-SPVD basis set. Counterpoise corrections were always included. As above, the assumed geometry is the result of taking carbon coordinates from crystal data, optimizing hydrogen coordinates with the M06-2X/def2-SPVD method. It is first noted the dramatic failure of mono-determinantal methods (Hartree-Fock) that cannot account for bonding in these systems (see also Fig. 2). In what concerns methods that include dispersion, note that those known to be the most accurate, MP4(SDTQ) and extrapolated $\operatorname{CCSD}(\mathrm{T})$, give results that differ at most in a $20 \%$, namely, 17.35 and $16.53 \mathrm{~kJ} / \mathrm{mol}$ for benzene, and 37.81 and $31.1 \mathrm{~kJ} / \mathrm{mol}$ for naphthalene, respectively. Those values are important as a reference for the less powerful, and less expensive, methods. In this sense, the results of Table S2 confirm the well-known overestimation of the dispersion energy that 2nd order perturbation methods (MP2) give ${ }^{61}$. However, although the subsequent improvement provided by MP3 strongly decreases $\Delta H_{s}^{t}$, when the highest order approximation is reached, i.e. MP4(SDTQ), it is substantially recovered. Actually, MP2 results are higher than those of MP4(SDTQ) in 20-30\%. The differences are slightly higher when compared with the best CC method. Results provided by the M06-2X method are quite close tho those of extrapolated $\operatorname{CCSD}(\mathrm{T})$ and around 10\% lower than those of the best MP4. However, improving the latter with the correction described above (M06-2X-D3) increases the values of $\Delta H_{s}^{t}$ above the results of both MP4 and CC methods. Anyhow, once considered these comments, we finally decide to calculate the sublimation enthalpies of all the crystals considered here by means of MP2, M06-2X and M06-2X-D3 methods.

\section{Complementary calculations}

Fig. S2 reports the electronic charge related to bonding (see Eq. 7) in anthracene (left) and tetracene (right) dimers calculated with the MP2/def2-TZVPP combination (uncorrelated charge density was calculated with the same basis set) to be compared with the same magnitudes calculated with the M06-2X/def2-TZVPP combination (see Fig. 4 of main text). The differences are merely quantitative.

Table S3 reports the same data as Table 1 but obtained with the smaller basis set def2SVPD, instead of def2-ZVTPP. In addition, this Table gathers the original experimental 
sublimation enthalpies (taken at room temperature) plus those corrected as explained above to shift the temperature down to $\mathrm{T}=0 \mathrm{~K}$ (temperature at which all calculations were done). The most remarkable conclusion reached when those two Tables are compared is the weak dependence of the MP2 results on the basis set, as compared to the significant changes that DFT results undergo when the smaller basis set is used.

Finally, the dependence of the SAPT results on geometry has been investigated comparing the results derived from geometries in which carbon coordinates have been taken from crystallographic data whereas hydrogen coordinates have been optimized by using either MP2/pc1 or M06-2X/def2-SVPD combinations. SAPT calculations were done with the 6$31 \mathrm{G}^{*}$ basis. The results are reported in Table $\mathrm{S} 4$. It can be readily noted that differences are almost negligible. We expect this result to be weakly dependent on which combinations are used. 


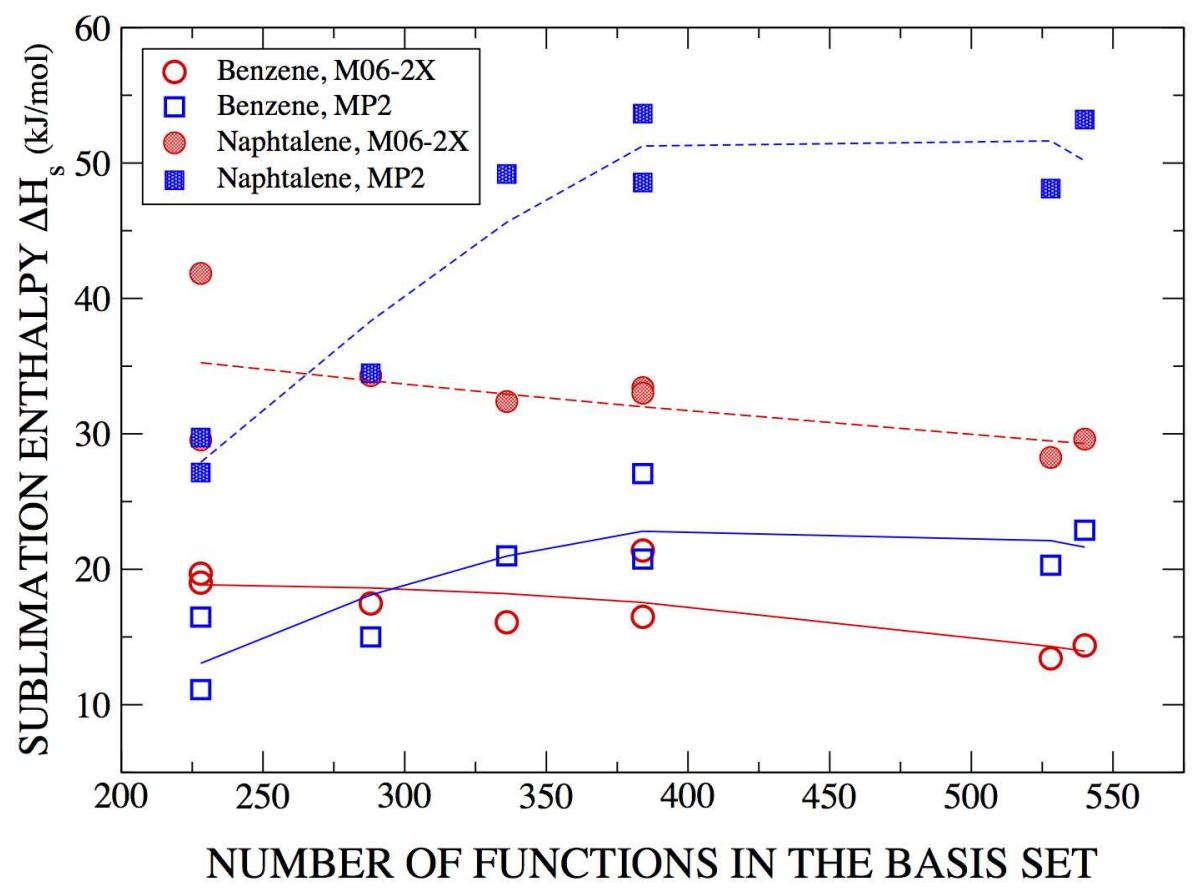

Figure S1: Sublimation enthalpies (in $\mathrm{kJ} / \mathrm{mol}$ ) of benzene and naphthalene calculated with M06-2X and MP2 methods versus the number of functions in the basis set. The numerical results are reported in Table $\mathrm{S} 1$. Lines are a guide to the eye.


Figure S2: Electronic charge related to bonding (see Eq. 7) in anthracene (left) and tetracene (right) dimers calculated by means of the MP2/def2-TZVPP combination (uncorrelated charge density was calculated with the same basis set). The geometry was determined by using the method proposed in this work, that is, carbons coordinates taken from crystallographic data and hydrogen coordinates optimized by means of the M06-2X/def2-SVPD combination. Blue color indicate positive sign, i.e., excess electronic density. 
Table S1: Numerical results for the enthalpy of sublimation $\left(\Delta H_{s}^{t}\right.$, in $\left.\mathrm{kJ} / \mathrm{mol}\right)$ calculated as twice the difference between the energy of two isolated molecules and that of the dimer (see Eq. (1)). Calculations were carried out using several basis sets and including the Counterpoise correction $(\mathrm{CP})$. The coordinates of the atoms in the dimer are the result of optimizing those of the hydrogens for each basis set while taking those of $\mathrm{C}$ atoms from crystallographic data; atom coordinates in the monomer were taken equal to those in the dimer. $\mathrm{N}_{B F}$ stands for the number of basis functions.

\begin{tabular}{cccccc}
\hline \hline & \multicolumn{3}{c}{ benzene } & \multicolumn{2}{c}{ naphthalene } \\
Basis set & $\mathrm{N}_{B F}$ & M06-2X & MP2 & M06-2X & MP2 \\
\hline 3-21G & 132 & 14.74 & 2.48 & 28.15 & -1.97 \\
6-311G** & 288 & 17.47 & 15.00 & 34.29 & 34.47 \\
cc-pVDZ & 228 & 19.67 & 16.47 & 29.55 & 29.73 \\
aug-cc-pVDZ & 384 & 21.38 & 27.06 & 33.42 & 53.64 \\
pc-1 & 228 & 19.02 & 11.11 & 41.85 & 27.14 \\
aug-pc-1 & 384 & 16.49 & 20.75 & 33.00 & 48.56 \\
pc-2 & 528 & 13.42 & 20.30 & 28.26 & 48.11 \\
def2-SVPD & 336 & 16.08 & 20.99 & 32.39 & 49.19 \\
def2-TZVPP & 540 & 14.37 & 22.88 & 29.61 & 53.21 \\
\hline \hline
\end{tabular}

Table S2: Enthalpy of sublimation $\left(\Delta H_{S}^{t}\right.$, in $\left.\mathrm{kJ} / \mathrm{mol}\right)$ of benzene and naphthalene calculated with Eq. (1), the def2-SPVD basis set and eleven methods. Counterpoise corrections were also included. Dimer geometry is the result of optimizing hydrogen coordinates with M062X/def2-SPVD method. The percentage of the experimental sublimation enthalpy $\Delta \mathrm{H}_{S}^{e}$ (see Table 1) covered by each theoretical calculation is given in the third and fifth columns. See also captions of previous Tables.

\begin{tabular}{ccccc}
\hline & \multicolumn{2}{c}{ benzene } & \multicolumn{2}{c}{ naphthalene } \\
Method & $\Delta H_{S}^{t}$ & $\% \Delta H_{S}^{e}$ & $\Delta H_{S}^{t}$ & $\% \Delta H_{S}^{e}$ \\
\hline HF & -16.05 & - & -47.21 & - \\
M06-2X & 16.08 & 32.68 & 32.39 & 44.61 \\
M06-2X-D3 & 19.70 & 40.04 & 40.38 & 55.61 \\
MP2 & 21.00 & 42.69 & 49.19 & 67.75 \\
MP3 & 10.87 & 22.09 & 12.51 & 17.24 \\
MP4(DQ) & 9.46 & 19.23 & 14.92 & 20.56 \\
MP4(SDQ) & 9.69 & 19.69 & 15.69 & 21.62 \\
MP4(SDTQ) & 17.35 & 35.27 & 37.81 & 52.08 \\
CCSD & 9.75 & 19.45 & 14.34 & 19.75 \\
CCSD(T) & 14.68 & 29.84 & 27.14 & 37.38 \\
extrapolated CCSD(T) & 16.53 & 33.60 & 31.10 & 42.84 \\
\hline
\end{tabular}


Table S3: Experimental data ${ }^{22}$ for the sublimation enthalpy $\Delta H_{S}^{e}$ (in $\mathrm{kJ} / \mathrm{mol}$ ) at room temperature (in parenthesis) and $\mathrm{T}=0 \mathrm{~K}$ (calculated using the results reported in Table 2) of some acenes, phenacenes and phenyls, and numerical results at $\mathrm{T}=0 \mathrm{~K}$ obtained using the def2-SVPD basis set and the methods MP2, M06-2X and M06-2X-D3. As concerns geometry, carbon coordinates were taken from crystal data whereas hydrogen coordinates were optimized using the M06-2X/def2-SPVD method. The percentage of the experimental data reached by the numerical results is also given at $\mathrm{T}=0 \mathrm{~K}$ and at room temperature (in parenthesis). The Counterpoise correction (CP) was also included.

\begin{tabular}{|c|c|c|c|c|c|c|c|c|}
\hline \multirow[b]{2}{*}{ Name } & \multirow[b]{2}{*}{ Formula } & \multirow[b]{2}{*}{$\mathrm{H}_{S}^{e}$} & \multicolumn{2}{|c|}{ M06-2X } & \multicolumn{2}{|c|}{ M06-2X-D3 } & \multicolumn{2}{|c|}{ MP2 } \\
\hline & & & $\Delta H_{s}^{t}$ & \%exp & $\Delta H_{s}^{t}$ & $\% \exp$ & $\Delta H_{s}^{t}$ & $\% \exp$ \\
\hline benzene & $\mathrm{C}_{6} \mathrm{H}_{6}$ & $\begin{array}{c}49.4 \\
(45.2 \pm 0.2)\end{array}$ & 16.08 & 32.6 & 19.70 & 39.9 & 21.00 & 42.5 \\
\hline naphthalene & $\mathrm{C}_{10} \mathrm{H}_{8}$ & $\begin{array}{c}76.9 \\
(72.6 \pm 0.3)\end{array}$ & 32.39 & 42.1 & 40.38 & 52.5 & 49.19 & 64.0 \\
\hline anthracene & $\mathrm{C}_{14} \mathrm{H}_{10}$ & $\begin{array}{c}105.6 \\
(101.9 \pm 1.3)\end{array}$ & 45.46 & 43.0 & 57.67 & 54.6 & 73.79 & 69.9 \\
\hline tetracene & $\mathrm{C}_{18} \mathrm{H}_{12}$ & $\begin{array}{c}139.9 \\
(135.9 \pm 5)\end{array}$ & 64.09 & 45.8 & 81.63 & 58.3 & 109.45 & 78.2 \\
\hline pentacene & $\mathrm{C}_{22} \mathrm{H}_{14}$ & $\begin{array}{c}152.9 \\
(148.9 \pm 4.2)\end{array}$ & 86.01 & 56.3 & 108.32 & 70.8 & 144.65 & 94.6 \\
\hline 3_phenacene & $\mathrm{C}_{14} \mathrm{H}_{10}$ & $\begin{array}{c}96.4 \\
(92.1 \pm 0.6)\end{array}$ & 40.53 & 42.1 & 52.76 & 30.8 & 66.82 & 69.3 \\
\hline 4_phenacene & $\mathrm{C}_{18} \mathrm{H}_{12}$ & $\begin{array}{c}124 \\
\left(120\left[{ }^{21}\right]\right)\end{array}$ & 59.13 & 47.7 & 76.24 & 61.5 & 97.59 & 78.7 \\
\hline 5_phenacene & $\mathrm{C}_{22} \mathrm{H}_{14}$ & $\begin{array}{c}153.4 \\
(149.4 \pm 2.9)\end{array}$ & 71.99 & 46.9 & 93.92 & 33.3 & 124.08 & 80.9 \\
\hline biphenyl & $\mathrm{C}_{12} \mathrm{H}_{10}$ & $\begin{array}{c}86.5 \\
(82.1 \pm 2.1)\end{array}$ & 42.60 & 49.2 & 53.33 & 61.6 & 61.94 & 71.6 \\
\hline p-terphenyl & $\mathrm{C}_{18} \mathrm{H}_{14}$ & $\begin{array}{c}130.1 \\
(125.6 \pm 1.6)\end{array}$ & 70.83 & 54.4 & 88.85 & 68.3 & 105.27 & 80.9 \\
\hline p-quaterphenyl & $\mathrm{C}_{24} \mathrm{H}_{18}$ & $\begin{array}{c}172.9 \\
(168.4 \pm 1.6)\end{array}$ & 97.49 & 56.4 & 122.92 & 71.1 & 147.83 & 85.5 \\
\hline
\end{tabular}


Table S4: Interaction energies (in $\mathrm{kJ} / \mathrm{mol}$ ) for acenes dimers calculated with the SAPT2/6$31 \mathrm{G}^{*}$ combination and using the geometries that result from taking carbon coordinates from crystal data and optimizing hydrogen coordinates with either MP2/pc1 or M06-2X/def2SVPD (in parenthesis) combinations. The exchange term contains first and second order corrections.

\begin{tabular}{lccccc}
\hline \hline System & $E_{\text {elec }}^{1}$ & $E_{\text {exch }}^{1+2}$ & $E_{\text {ind }}^{2}$ & $E_{\text {disp }}^{2}$ & $E_{\text {int }}^{\text {SAPT }}$ \\
\hline benzene & $-9.508(-9.490)$ & $21.428(21.508)$ & $-1.030(-1.031)$ & $-14.243(-14.270)$ & $-3.354(-3.283)$ \\
naphthalene & $-22.789(-23.062)$ & $52.398(53.019)$ & $-2.089(-2.126)$ & $-34.399(-34.599)$ & $-6.879(-6.768)$ \\
anthracene & $-15.353(-15.354)$ & $32.548(32.548)$ & $-1.989(-1.990)$ & $-34.428(-34.427)$ & $-19.222(-19.223)$ \\
tetracene & $-23.053(-23.053)$ & $50.543(50.544)$ & $-3.040(-3.041)$ & $-52.297(-52.298)$ & $-27.851(-27.849)$ \\
pentacene & $-30.392(-30.393)$ & $64.941(64.941)$ & $-4.534(-4.532)$ & $-71.150(-71.153)$ & $-41.135(-41.137)$ \\
\hline \hline
\end{tabular}

